\title{
Presence of Propionibacterium acnes in granulomas associates with a chronic disease course in Dutch sarcoidosis patients
}

\author{
Els Beijer (10 ${ }^{1}$, Kees Seldenrijk² ${ }^{2}$, Yoshinobu Eishi $\mathbb{1}^{3}$, Keisuke Uchida ${ }^{3}$, \\ Jan Damen ${ }^{4}$, Jan C. Grutters ${ }^{1,5}$ and Marcel Veltkamp ${ }^{1,5}$
}

Affiliations: ${ }^{1}$ Interstitial Lung Diseases Centre of Excellence, Dept of Pulmonology, St Antonius Hospital, Nieuwegein, The Netherlands. ${ }^{2}$ Pathology DNA, Dept of Pathology, St Antonius Hospital, Nieuwegein, The Netherlands. ${ }^{3}$ Dept of Human Pathology, Tokyo Medical and Dental University, Tokyo, Japan. ${ }^{4}$ Pathology DNA Dept of Pathology, Jeroen Bosch Hospital, 's-Hertogenbosch, The Netherlands. ${ }^{5}$ Division of Heart and Lungs, University Medical Centre, Utrecht, The Netherlands.

Correspondence: Marcel Veltkamp, ILD research, Koekoekslaan 1, 3435 CM Nieuwegein, The Netherlands. E-mail: m.veltkampdaantoniusziekenhuis.nl

ABSTRACT Several studies demonstrated that Propionibacterium acnes may be involved in sarcoidosis pathogenesis. Presence of $P$. acnes was found in granulomas of the majority of Japanese sarcoidosis patients. However, presence of $P$. acnes in tissue has never been related to sarcoidosis phenotypes and clinical outcome. Therefore, the aims of our study were to demonstrate whether P. acnes can be detected in granulomas of Dutch sarcoidosis patients and to investigate whether its presence is related to a clinical phenotype and/or course of disease.

Sections of formalin-fixed paraffin-embedded tissue blocks of 76 sarcoidosis patients were examined by immunostaining with a $P$. acnes-specific monoclonal antibody (PAB antibody) using a Ventana BenchMark ULTRA. Clinical outcome status (COS) was determined and classified into two phenotype groups: A: resolved, minimal or persistent disease without treatment (COS 1-6) and B: persistent disease with need for treatment (COS 7-9).

P. acnes was detected in samples of 31 patients (41\%) and located within granulomas in samples of 13 patients $(17 \%)$. The frequency of $P$. acnes detected in granulomas at diagnosis was significantly higher in patients with phenotype B compared to patients with phenotype A ( $29 \%$ versus $0 \%, \mathrm{p}=0.021)$.

Presence of $P$. acnes in granulomas can be confirmed in Dutch sarcoidosis patients. It is intriguing that presence of $P$. acnes in granulomas is more frequently found in patients with chronic disease requiring treatment. This adds to the rationale that a subgroup of sarcoidosis patients might benefit from antibiotic therapy.

@ERSpublications

Significantly more sarcoidosis patients with a chronic disease course requiring treatment have presence of $P$. acnes in granulomas. This contributes to the premise that it is relevant to further explore antibacterial therapy in sarcoidosis. https://bit.ly/36wCbbw

Cite this article as: Beijer E, Seldenrijk K, Eishi Y, et al. Presence of Propionibacterium acnes in granulomas associates with a chronic disease course in Dutch sarcoidosis patients. ERJ Open Res 2021; 7: 00486-2020 [https://doi.org/10.1183/23120541.00486-2020].

Received: 10 July 2020 | Accepted after revision: 6 Nov 2020

Copyright $\odot$ ERS 2021. This article is open access and distributed under the terms of the Creative Commons Attribution Non-Commercial Licence 4.0. 


\section{Introduction}

Sarcoidosis is a multisystem inflammatory disorder of unknown aetiology that is characterised by the presence of noncaseating granulomas. In over $90 \%$ of patients the lungs are involved [1], but the disease can also affect other organs [2]. Patients with symptomatic organ involvement or risk of permanent damage can be treated with immunosuppressive drugs [3], which suppress disease activity but do not cure the disease.

The cause of sarcoidosis is still puzzling scientists for more than a century. Several studies demonstrated that specific microorganisms, including mycobacteria and Propionibacterium acnes could be involved in its disease pathogenesis [4-9]. As P. acnes is a commensal, Koch's postulates cannot be applied, which makes it difficult to elucidate its aetiological role [10]. A higher number of $P$. acnes genomes have been found in tissue from Chinese, Japanese, Italian, English and German sarcoidosis patients compared to tissue of controls, suggesting involvement in disease pathogenesis [11-13]. Furthermore, an increased immune response to $P$. acnes among patients with sarcoidosis was found in different studies using Japanese or German patients $[7,8,14]$. Remarkably, in recent work from our own group, we found a lower percentage of Dutch sarcoidosis patients than controls with an immunological response to P. acnes [15], which is conflicting with the above-mentioned papers.

In addition to its role as possible antigen, $P$. acnes can also act as a mitogen, which is demonstrated by the fact that $P$. acnes enhances immunogenicity of certain vaccines [16] and enhances cytotoxic activity toward different tumour cells [17-19]. A potential mitogenic role of $P$. acnes in sarcoidosis has not previously been studied.

Based on both a possible antigenic as well as mitogenic role for P. acnes, we hypothesise that presence of $P$. acnes in tissue could be related to certain clinical phenotypes in sarcoidosis. We therefore examined the presence of $P$. acnes in tissue of Dutch patients with sarcoidosis using an existing $P$. acnes-specific monoclonal antibody (PAB antibody) [20] and related results of immunostaining to clinical characteristics such as organ involvement and course of disease.

\section{Methods and materials}

\section{Study patients}

Unstained tissue blocks were requested from two sarcoidosis cohorts previously studied at the St Antonius Hospital (Nieuwegein, the Netherlands) [15, 21]. The diagnosis of sarcoidosis had been established according to the criteria of the American Thoracic Society/European Respiratory Society [22]. Patients were included in the study when enough residual tissue was available and when presence of granulomas could be clearly detected in the haematoxylin and eosin (H\&E) stained tissue sections. The study was approved by the Medical Research Ethics Committees United (MEC-U) of the St Antonius Hospital (R05-08A) and written consent was obtained from all patients.

\section{Immunostaining}

$4-\mu \mathrm{m}$ thick sections were cut from the formalin-fixed paraffin-embedded tissue sections, which were immunohistochemically stained with the PAB antibody; a $P$. acnes-specific monoclonal antibody that reacts with cell membrane-bound lipoteichoic acid (LTA) of the bacterium [20]. The PAB antibody was kindly provided by Y. Eishi and colleagues (Department of Human Pathology, Tokyo Medical and Dental University, Tokyo, Japan). We followed the protocol described by NEGI et al. [20], but instead of the original manual procedures the sections were stained by the use of a Ventana BenchMark ULTRA (Ventana Medical Systems Inc., Tucson, AZ, USA) using an ultraView Universal Alkaline Phosphatase Red Detection kit (Ventana Medical Systems). We modified the original protocol to optimise the sensitivity and specificity of the staining results for the Ventana BenchMark ULTRA. Shortly, sections were de-paraffinised and rehydrated followed by antigen retrieval using the hot plate heating system of the machine instead of antigen retrieval by microwave. Because mineral oil (Liquid Coverslip, Ventana Medical Systems, Inc.) covering the tissue slides was found to inhibit the reaction with PAB antibody, before the primary antibody reaction with the PAB antibody, a washing step (three times for 5 min each) in the EZ Prep buffer (Roche Nederland B.V., Woerden, the Netherlands) was added under the Antibody Titration program selected, followed by washing for $5 \mathrm{~min}$ with tap water and $5 \mathrm{~min}$ with reaction buffer (Roche Nederland B.V.). The PAB antibody (crude mouse ascites fluid) was used in a concentration of 1:30 000 (diluted with DAKO REAL antibody diluent, S2022, DAKO, Glostrup, Denmark) and incubated for $16 \mathrm{~min}$ at room temperature. Instead of using the peroxidase substrate diaminobenzidine (DAB) to develop the signal, an ultraView Universal Alkaline Phosphatase Red Detection kit (Ventana Medical Systems) was used. Sections were counterstained with Mayer's haematoxylin. Detection of P. acnes in tissue was analysed by a pulmonary pathologist (K. Seldenrijk). The staining was considered positive when 
small round/dot-like structures were seen. If such structures were detected, it was determined whether positive $\mathrm{PAB}$ staining was present in or outside the granulomas.

\section{Identification of P. acnes-related phenotypes}

To determine possible $P$. acnes-related phenotypes, organ involvement, age at diagnosis and Scadding stage at diagnosis and follow-up was collected from medical records of sarcoidosis patients.

The clinical outcome status (COS), a definition of clinical outcome in sarcoidosis established by the World Association of Sarcoidosis and Other Granulomatous Diseases [23]was determined 2 and 5 years after diagnosis. The disease status of patients was retrospectively examined and classified into resolved, minimal or persistent disease. Resolved was classified as patients showing no signs of disease, such as normalisation of chest radiography, pulmonary function tests and laboratory tests. Minimal disease is defined as a disease burden of $25 \%$ or less compared with the maximum disease burden experienced by patients measured by pulmonary function tests, chest radiography, biomarkers and skin lesions. For example, the worst pulmonary function test had to be improved by at least $75 \%$ to be considered minimal disease.

Furthermore, it was examined whether patients were ever treated, and if so whether they were still using medication or not (defined as no medication $>1$ year). Patients in whom medication was increased the last year were considered as worsening. Medication included all immunosuppressive systemic therapies used for sarcoidosis, including corticosteroids, disease-modifying antirheumatic drugs and anti-tumour necrosis factor (TNF)- $\alpha$ antibodies, except for nonsteroidal anti-inflammatory drugs. This results in nine COS scores. 1: Resolved disease, never treated; 2: resolved diseases, no treatment $>1$ year; 3: minimal disease, never treated; 4: minimal disease, no therapy >1 year; 5: persistent disease, never treated; 6: persistent disease, no therapy $>1$ year; 7 : persistent disease, current therapy but no worsening in prior year and asymptomatic; 8: persistent disease, current therapy but no worsening in prior year and symptomatic; and 9: persistent disease, current therapy that worsened in the prior year.

Patients who died in the period over which the COS was determined were placed in COS 9.

To analyse whether there was a correlation between presence of $P$. acnes and COS, we classified the COS scores into two phenotype groups: A: resolved, minimal or persistent disease without treatment (COS 1-6) and B: persistent disease with need for treatment (COS 7-9).

\section{Statistical analysis}

Data were analysed using IBM SPSS statistics version 24. An unpaired t-test was used to compare numerical data. Nonparametric tests were used for non-normally distributed data (Mann-Whitney U-test). Categorical data were compared using the Chi-squared test. If expected cell frequencies were below 5, Fisher's exact test was used for categorical data up to two categories. A p-value $<0.05$ was considered significant.

\section{Results}

\section{Characteristics of study patients and tissue samples}

Formalin-fixed paraffin-embedded tissue blocks were available from 76 patients. Mean age of included patients was 44 years, $84 \%$ were white and $71 \%$ had extrapulmonary involvement. Overall, $68 \%$ and $67 \%$ were classified in phenotype group B 2 and 5 years after diagnosis, respectively (table 1).

\section{P. acnes can be detected in tissue samples of Dutch patients with sarcoidosis}

Most tissue sections used for staining originated from the lung, followed by lymph node and skin samples. $P$. acnes was detected in tissue samples from 31 of the 76 sarcoidosis patients (41\%) and was located in the granulomas in 13 samples of all patients (17\%) (table 2 and figs. 1 and 2). When P. acnes was not detected inside granulomas, it was mostly located directly adjacent to granulomas in histiocytes and in a few cases in granuloma-free areas of the tissue. Besides lung, lymph node and skin tissue, we also found presence of $P$. acnes in bone marrow and liver tissue. No significant difference was observed in the frequency of $P$. acnes detected in tissue or granulomas between the different organs used in the study $(\mathrm{p}=0.583$ and $\mathrm{p}=0.490$, respectively). P. acnes was detected in tissue or granulomas in three (43\%) and three $(43 \%)$, respectively of the seven lung samples obtained by video-assisted thoracic surgery (VATS), and in seven (28\%) and four (16\%), respectively of the lung samples obtained by transbronchial lung biopsy (TBLB). No significant difference in presence of $P$. acnes in tissue or granulomas was observed between the different biopsy methods used for the lung samples ( $\mathrm{p}=0.648$ and $\mathrm{p}=0.157$, respectively) and lymph node samples $(\mathrm{p}=0.167$ and $\mathrm{p}=0.588$, respectively). 


\section{TABLE 1 Characteristics of study patients}

Sarcoidosis $(\mathrm{n}=76$

\begin{tabular}{|c|c|}
\hline Age at time of biopsy years & $43.98 \pm 12.22$ \\
\hline Male sex & 42 (55) \\
\hline Ever smoker & $43(59)$ \\
\hline White & $64(84)$ \\
\hline Medication at time of biopsy & $6(8)$ \\
\hline Third-line therapy ${ }^{\#}$ & $27(36)$ \\
\hline COS group A/B (2 years follow-up) & $23 / 49$ (32/68) \\
\hline $\operatorname{COS}$ group A/B (5 years follow-up) & $15 / 31(33 / 67)$ \\
\hline Scadding stage at time biopsy (0/I/II/III/IV/unknown) & $5 / 19 / 28 / 9 / 10 / 5(7 / 25 / 37 / 12 / 13 / 7)$ \\
\hline Extrapulmonary involvement & $54(71)$ \\
\hline Skin & $18(24)$ \\
\hline Eyes & 7 (9) \\
\hline Liver & $8(11)$ \\
\hline Heart & $13(17)$ \\
\hline Spleen & $3(4)$ \\
\hline Bones & $4(5)$ \\
\hline Nerve system & $15(20)$ \\
\hline Central & $6(8)$ \\
\hline Peripheral & $1(1)$ \\
\hline Small fibre neuropathy & $10(13)$ \\
\hline
\end{tabular}

Presence of $\mathrm{P}$. acnes does not correlate with organ involvement

When relating positive $P$. acnes staining results to clinical characteristics, no relation with age, sex, ethnicity, organ involvement or Scadding stage was observed (table 3).

\section{Presence of P. acnes correlates with clinical outcome}

Presence of $P$. acnes in tissue was compared between the two COS groups defined 2 and 5 years after diagnosis. Two years after diagnosis, a trend $(\mathrm{p}=0.093)$ towards a higher prevalence of $P$. acnes in tissue was seen in COS group B (47\%, 23 of 49) compared to COS group A (26\%, 6 of 23). When comparing presence of $P$. acnes specifically inside granulomas, also a trend $(\mathrm{p}=0.050)$ towards a higher prevalence was seen in COS group B patients $(25 \%, 12$ of 49$)$ compared to COS group A patients $(4 \%, 1$ of 23 ) (fig. 3a). COS defined after 5 years revealed a trend $(\mathrm{p}=0.072)$ towards a higher prevalence of $P$. acnes in tissue in COS group B (55\%, 17 of 31$)$ compared to COS group A (27\%, 4 of 15$)$. A significantly higher percentage of patients within COS group B $(29 \%, 9$ of 31$)$ were positively stained for P. acnes inside granulomas compared to COS group A patients $(0,0$ of 15$)$ ( $p=0.021$, fig. $3 b)$.

As COS group B can be a very heterogeneous group of patients, ranging from patients with remission of symptoms due to long-term corticosteroid treatment to severe therapy-refractory patients requiring third-line medication, we also determined whether presence of $P$. acnes in tissue and granulomas was specifically related to patients requiring third-line medication at follow-up. In a significantly higher percentage of patients using infliximab, $P$. acnes was present in granulomas compared to patients not requiring third-line therapy ( $33 \%$ versus $8 \%, \mathrm{p}=0.009)$ (fig. 4 ).

\section{Discussion}

In this study we demonstrated that $P$. acnes is present in granulomas of Dutch patients with sarcoidosis, which is in line with previous studies in Japanese and German sarcoidosis patients [20]. Furthermore, to the best of our knowledge, our study is the first to show a relation between presence of $P$. acnes and clinical outcome in sarcoidosis. Regardless of whether the role of $P$. acnes might be antigenic or mitogenic, these data add to the rationale that $P$. acnes might be involved in the disease pathogenesis of sarcoidosis.

Negr et al. [20] previously examined the presence of $P$. acnes in tissue samples from sarcoidosis patients, using the same $\mathrm{PAB}$ antibody as used in the current study. A remarkable difference was found in percentage of $P$. acnes-positive sarcoidosis patients. They found $P$. acnes in granulomas in $57 \%$ of the 77 lung samples from Japanese sarcoidosis patients, whereas we found $P$. acnes in granulomas in $22 \%$ of the 32 lung samples from Dutch sarcoidosis patients. This difference could be partially explained by the lower 


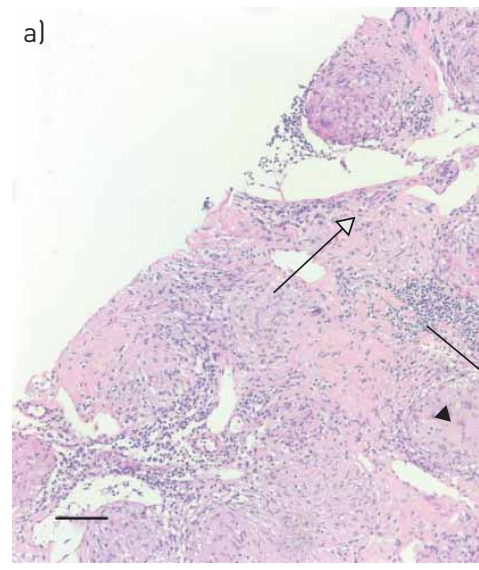

c)

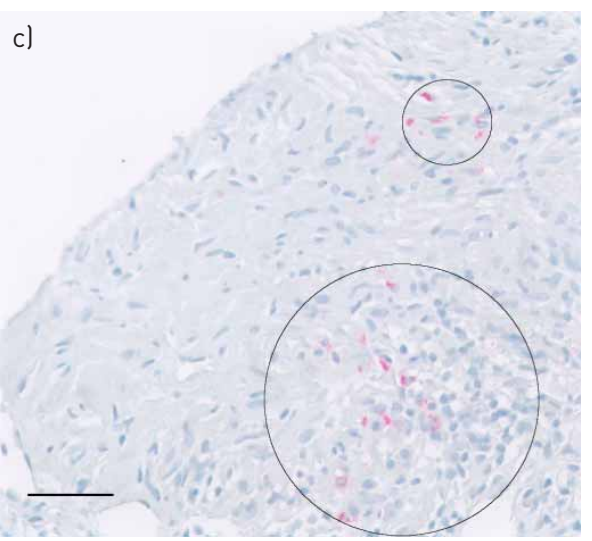

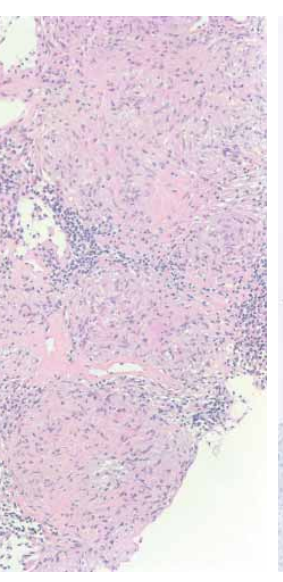

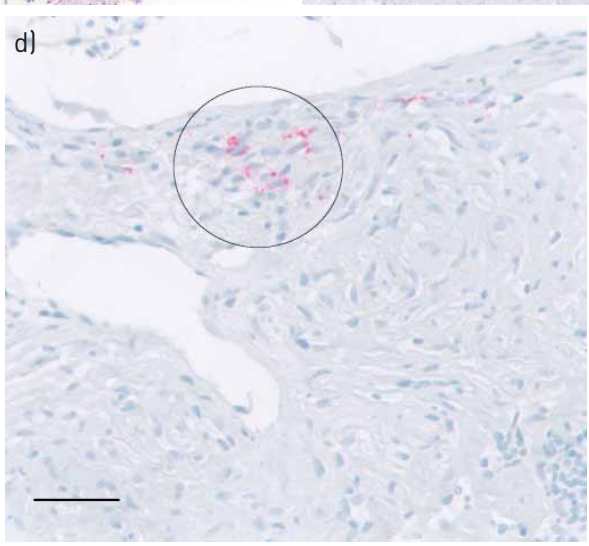

b)
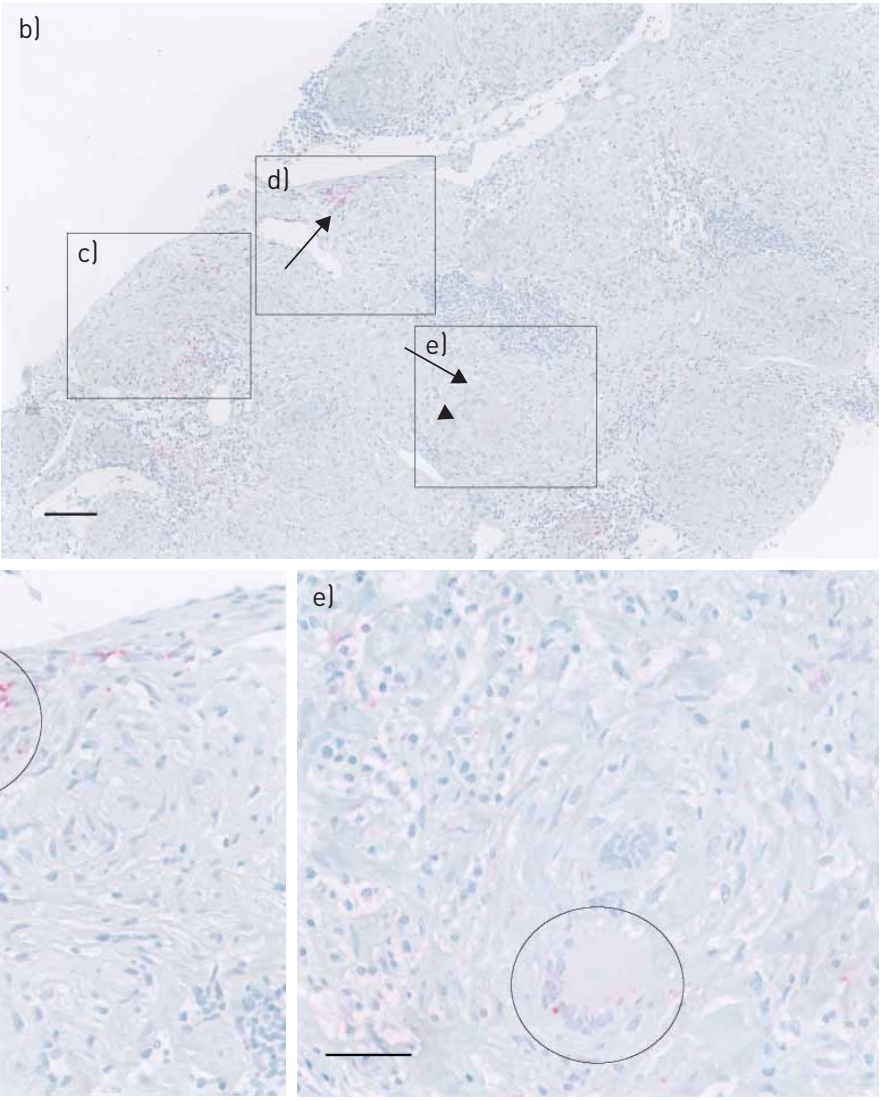

FIGURE 1 Presence of Propionibacterium acnes in and around an immature granuloma of the lymph node. a) Haematoxylin and eosin (H\&E)

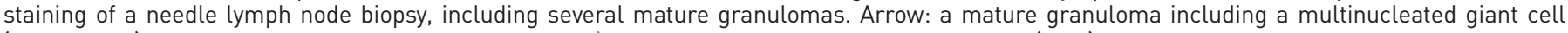
(arrow head). Open arrow: an immature granuloma. b) $P$. acnes-specific monoclonal antibody (PAB) staining corresponding with the area in a, including several areas with $P$. acnes-positive red signals ( $c$ and $d$ ) and a mature granuloma negative for $P$. acnes ( ). $c$ ) Higher magnification of positive $P$. acnes staining (circles) around granulomas. d) Higher magnification of positive $P$. acnes staining (circle) in an immature granuloma. e) Higher magnification of another area including a multinucleated giant cell positive for $P$. acnes. Scale bars=100 $\mu \mathrm{m}$ (a and b) and $50 \mu \mathrm{m}$ (c-e)

proportion of VATS samples (35\% in their study compared to $22 \%$ in our study). NeGI et al. [20] described that $P$. acnes in granulomas was more frequently detected in VATS samples compared to TBLB samples, probably due to the larger size of VATS samples. Similar to the lung samples, the percentage of Dutch patients with presence of $P$. acnes in granulomas of lymph node samples (8\%) was lower compared to Japanese and German patients ( $88 \%$ and $89 \%$, respectively). Although the difference between Japanese and Dutch patients may be explained by difference in ethnicity, this is not a plausible explanation for the difference between German and Dutch patients. The difference in the detection frequency of $P$. acnes in granulomas can possible be caused by a lower sensitivity of immunostaining method used in our study. Therefore, future studies will have to compare the detection sensitivity of $P$. acnes in granulomas using the different immunostaining methods. It is however important to state that the localisation and pattern of $P$. acnes within and outside granulomas was comparable with the study of NEGI et al. [20]. Specifically, we observed that presence of $P$. acnes outside granulomas was most frequently detected directly adjacent to granulomas but also in granuloma-free parts of the tissue in a few cases. Furthermore, the pattern within granulomas was comparable, with more intense dot-like structures in immature granulomas and more sparsely distributed staining or even no staining in mature granulomas.

A new and clinically relevant finding, in our opinion, is the fact that this is the first study that shows an association between presence of $P$. acnes and clinical outcome in patients with sarcoidosis. We observed that presence of $P$. acnes in tissue and in granulomas is more frequently found in patients with a chronic disease course requiring chronic treatment. Several studies demonstrated the mitogenic properties of $P$. acnes on different immune cells $[16,24,25]$. Our study was not aimed to prove that $P$. acnes has a specific aetiological role in sarcoidosis; however, in our opinion, the results do suggest that at least a potential mitogenic role of $P$. acnes may contribute to sarcoidosis disease pathogenesis. It is tempting to speculate that presence of $P$. acnes in or around granulomas can enhance an ongoing inflammatory reaction in sarcoidosis, contributing to perpetuation of the inflammatory granulomatous response seen in some 

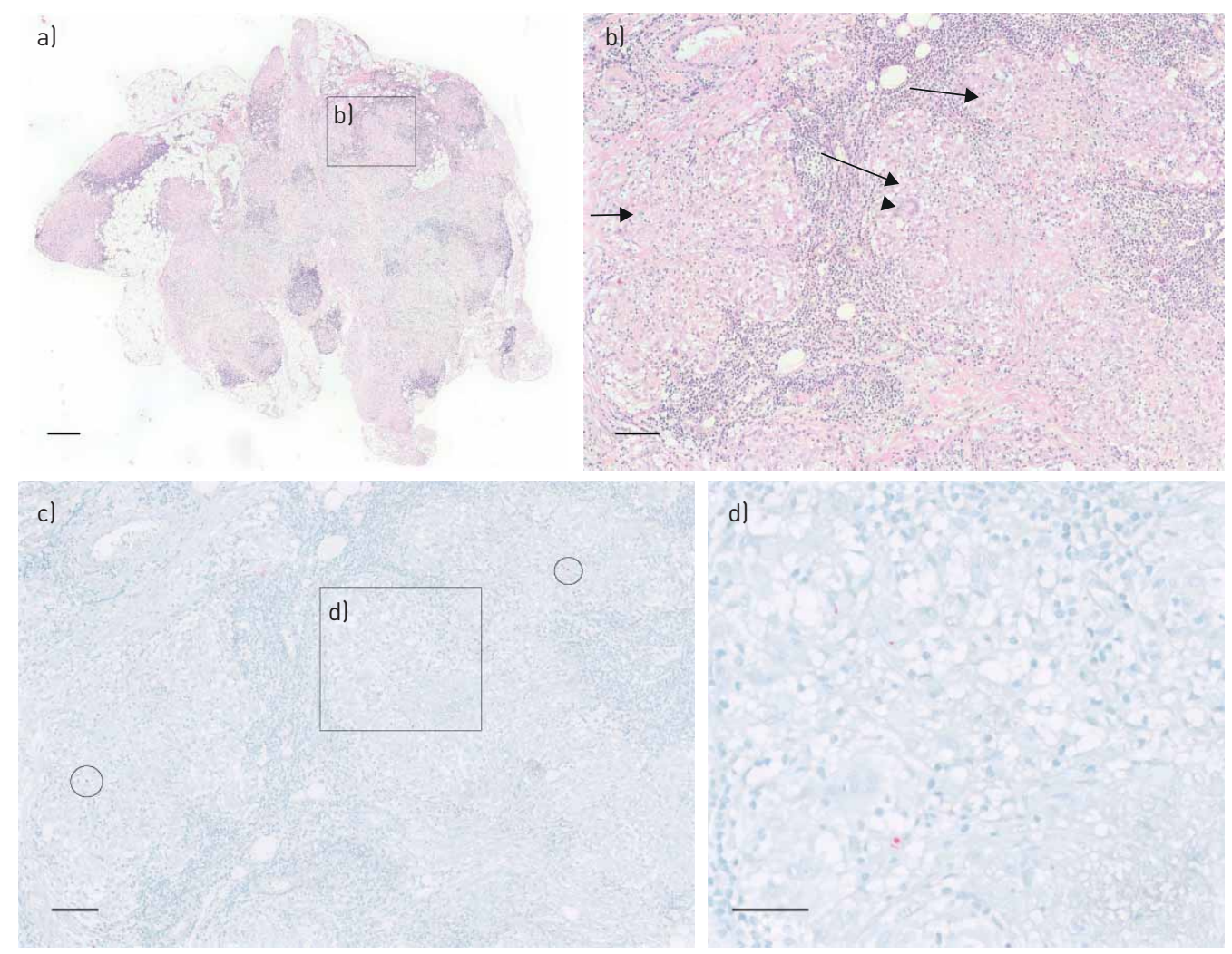

FIGURE 2 Presence of Propionibacterium acnes in a mature granuloma in s.c. tissue of the lower eyelid. a) Overview of haematoxylin and eosin (H\&E) staining of s.c. tissue of the lower eyelid. b) Higher magnification of area $b$ from picture $a$, including mature granulomas (arrows) and multinucleated giant cells (arrow head). c) P. acnes-specific monoclonal antibody (PAB) staining of corresponding area of $b$, including several areas of $P$. acnes-positive signals (circles and rectangle d), in mature granulomas. d) Higher magnification of area $d$ from picture $c$, with positive $P$. acnes staining in a mature granuloma. Scale bars=500 $\mu \mathrm{m}(\mathrm{a}), 100 \mu \mathrm{m}(\mathrm{b}, \mathrm{c})$ and $50 \mu \mathrm{m}$ (d)

patients. If this holds true, it is interesting to see whether decreasing the bacterial load of $P$. acnes using antibiotics can be beneficial in a subgroup of patients with sarcoidosis.

A case report already described a good effect of clarithromycin on fever, joint pain, 2-fluoro-2-deoxy-D-glucose uptake on positron emission tomography/computed tomography, C-reactive protein and s-interleukin-2R levels in a sarcoidosis patient in whom $P$. acnes was present in granulomas [26]. Moreover, a retrospective study described a good response on treatment with minocycline in P. acnes-positive cutaneous sarcoidosis patients [27]. When we examined severe therapy-refractory patients
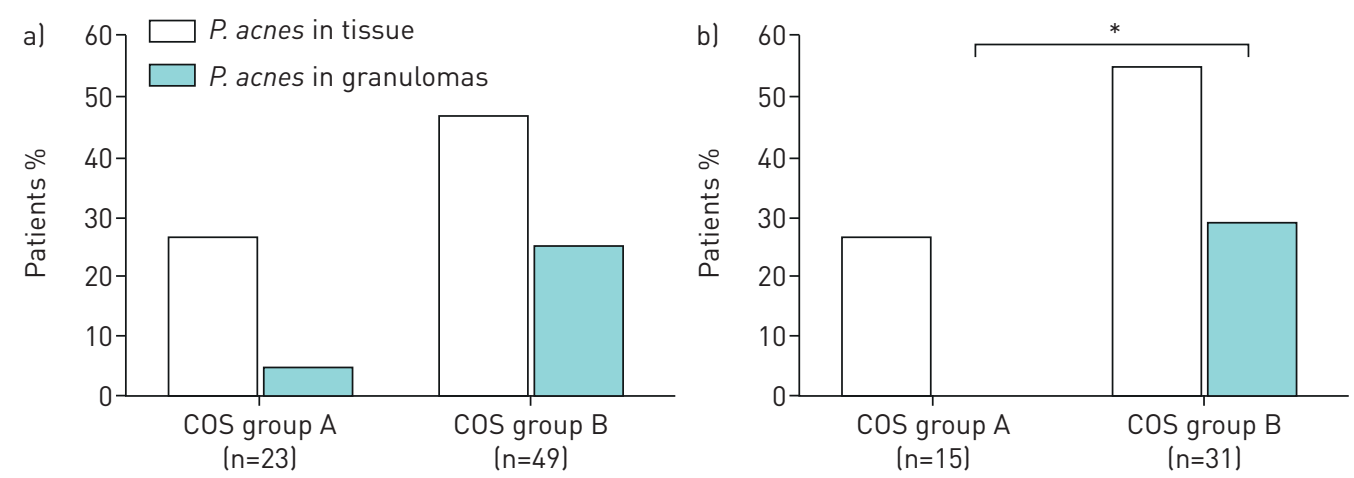

FIGURE 3 Frequency of Propionibacterium acnes detected in tissue samples from sarcoidosis patients with a different clinical outcome status (COS) 2 years (a) and 5 years (b) after diagnosis. COS group A indicates resolved, minimal or persistent disease without treatment (COS 1-6). COS group B indicates persistent disease with need for treatment (COS 7-9). PAB: P. acnes-specific monoclonal antibodies that react with cell membrane-bound lipoteichoic acid. 


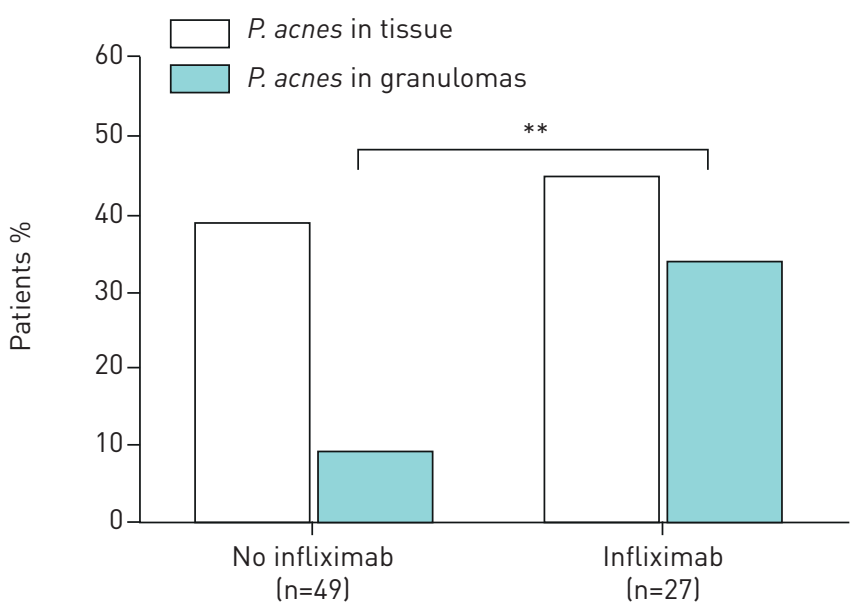

FIGURE 4 Frequency of Propionibacterium acnes detected in biopsy samples from sarcoidosis patients with or without third-line therapy (infliximab) during follow-up. A significantly higher percentage of third-line therapy-requiring patients (all using infliximab) showed presence of $P$. acnes in granulomas than patients who did not require third-line therapy $(p=0.009)$. PAB: $P$. acnes-specific monoclonal anti-bodies that react with cell membrane-bound lipoteichoic acid.

for the presence of $P$. acnes, we observed that patients requiring infliximab treatment at follow-up, often had more presence of $P$. acnes in granulomas at diagnosis compared to patients without treatment or treated with first- or second-line treatment. If antibacterial treatment in sarcoidosis patients with presence of $P$. acnes in tissue and granulomas is beneficial, the requirement of third-line treatments (e.g. infliximab) may be prevented.

Currently, in a randomised controlled clinical trial (J-ACNES), the effect of antibacterial drugs in addition to standard corticosteroid therapy in cardiac sarcoidosis patients is examined [28]. However, in this trial presence of $P$. acnes in myocardial tissue was not an inclusion criterion. Therefore, in future studies it would be interesting to have information on the presence or absence of $P$. acnes in these patients, while investigating the effect of antibiotic therapy.

\section{TABLE 2 Detection of Propionibacterium acnes and origin of tissue used}

\begin{tabular}{|c|c|c|c|}
\hline & \multirow[t]{2}{*}{$\mathbf{n}$} & \multicolumn{2}{|c|}{ Detection of $P$. acnes in: } \\
\hline & & Tissue & Granulomas \\
\hline Total patients ${ }^{\#}$ & 76 & $31(41)$ & $13(17)$ \\
\hline Total tissue sections & 80 & $32(40)$ & 14 (18) \\
\hline Lung & 32 & $10(31)$ & $7(22)$ \\
\hline VATS & 7 & $3(43)$ & $3(43)$ \\
\hline TBLB & 25 & $7(28)$ & $4(16)$ \\
\hline Lymph node & 25 & $12(48)$ & $2(8)$ \\
\hline Lymphadenectomy & 14 & $9(64)$ & $1(7)$ \\
\hline Mediastinoscopy & 5 & $1(20)$ & 0 \\
\hline EBUS-TBNA/needle & 6 & $2(33)$ & $1(17)$ \\
\hline Skin & 17 & $7(41)$ & $4(24)$ \\
\hline Other & 6 & $3(50)$ & $1(17)$ \\
\hline Bone marrow & 2 & $2(100)$ & 0 \\
\hline Liver & 2 & $1(50)$ & $1(50)$ \\
\hline Nasal concha & 1 & 0 & 0 \\
\hline Salivary gland & 1 & 0 & 0 \\
\hline \multicolumn{4}{|c|}{$\begin{array}{l}\text { Data are presented as } \mathrm{n}(\%), \text { unless otherwise stated. \#: In } 4 \text { out of } 76 \text { patients, two tissue sections of } \\
\text { different organs were stained namely: lymph node (negative) and lung ( } P \text {. acnes-positive in tissue and } \\
\text { granulomas), liver (negative) and lymph node (P. acnes-positive in tissue), skin (P. acnes-positive in tissue } \\
\text { and granulomas) and lung (negative), skin ( } P \text {. acnes-positive in tissue and granulomas) and lung } \\
\text { (P. acnes-positive in tissue and granulomas). VATS: video-assisted thoracoscopic surgery; TBLB: } \\
\text { transbronchial lung biopsy; EBUS-TBNA: endobronchial ultrasound-transbronchial needle aspiration. }\end{array}$} \\
\hline
\end{tabular}




\begin{tabular}{|c|c|c|c|c|c|c|}
\hline & \multicolumn{3}{|c|}{ PAB staining } & \multicolumn{3}{|c|}{ PAB staining present in granulomas } \\
\hline & $\begin{array}{r}\text { Negative } \\
(n=45)\end{array}$ & $\begin{array}{l}\text { Positive } \\
\quad(n=31)\end{array}$ & p-value ${ }^{\#}$ & No $(n=63)$ & Yes $(n=13)$ & p-value ${ }^{\#}$ \\
\hline Age $^{\text {ๆ }}$ years & $44.59 \pm 12.51$ & $43.08 \pm 11.94$ & 0.601 & $44.76 \pm 12.17$ & $40.18 \pm 12.26$ & 0.222 \\
\hline Male sex & $23(51)$ & $19(61)$ & 0.380 & $33(52)$ & 9 (69) & 0.266 \\
\hline White & $38(84)$ & $26(84)$ & 1.000 & $52(83)$ & 12 (92) & 0.679 \\
\hline $\begin{array}{l}\text { Extrapulmonary } \\
\text { involvement }\end{array}$ & $32(71)$ & $22(71)$ & 0.989 & $46(73)$ & $8(62)$ & 0.504 \\
\hline Skin & $12(27)$ & $6(19)$ & 0.461 & $15(24)$ & $3(23)$ & 1.000 \\
\hline Eyes & 4 (9) & $3(10)$ & 1.000 & $6(10)$ & $1(8)$ & 1.000 \\
\hline Liver & $2(4)$ & $6(19)$ & 0.057 & $6(10)$ & $2(15)$ & 0.619 \\
\hline Heart & $8(18)$ & $5(16)$ & 0.851 & $12(19)$ & $1(8)$ & 0.446 \\
\hline Spleen & $2(4)$ & $1(3)$ & 1.000 & 2 (3) & 1 (8) & 0.435 \\
\hline Bones & $3(7)$ & $1(3)$ & 0.641 & $4(6)$ & 0 & 1.000 \\
\hline Nerve system & $11(24)$ & $4(13)$ & 0.214 & $14(22)$ & 1 (8) & 0.444 \\
\hline Central & $5(11)$ & $1(3)$ & 0.391 & $6(10)$ & 0 & 0.582 \\
\hline Peripheral & $1(2)$ & 0 & 1.000 & $1(2)$ & 0 & 1.000 \\
\hline SFN & $7(16)$ & $3(10)$ & 0.514 & 9 (14) & 1 (8) & 1.000 \\
\hline $\begin{array}{l}\text { Scadding stage at } \\
\text { diagnosis (0/I/ } \\
\text { II/III/IV) }\end{array}$ & $\begin{array}{c}(\mathrm{N}=44), 3 / 13 / \\
19 / 5 / 4(7 / 30 / \\
43 / 11 / 9)\end{array}$ & $\begin{array}{c}(N=29), 0 / 10 / \\
13 / 2 / 4(0 / 35 / \\
45 / 7 / 14)\end{array}$ & 0.585 & $\begin{array}{c}(\mathrm{N}=61), 3 / 19 / \\
29 / 5 / 5(5 / 31 / \\
48 / 8 / 8)\end{array}$ & $\begin{array}{c}(\mathrm{N}=12), 0 / 4 / \\
3 / 2 / 3(0 / 33 / \\
25 / 17 / 25)\end{array}$ & 0.277 \\
\hline $\begin{array}{l}\text { Scadding stage } \\
\text { after } 2 \text { years (0/ } \\
\text { I/II/III/IV) }\end{array}$ & $\begin{array}{c}(\mathrm{N}=39), 11 / 4) \\
12 / 5 / 7(28 / \\
10 / 31 / 13 / 18)\end{array}$ & $\begin{array}{c}(N=24), 6 / 3 / \\
8 / 1 / 6(25 / 13 / \\
33 / 4 / 25)\end{array}$ & 0.794 & $\begin{array}{c}(N=53), 14 / 6 / \\
19 / 5 / 9(26 / \\
11 / 36 / 9 / 17)\end{array}$ & $\begin{array}{c}(\mathrm{N}=10), 3 / 1 / \\
1 / 1 / 4(30 / 10 / \\
10 / 10 / 40)\end{array}$ & 0.408 \\
\hline $\begin{array}{l}\text { Scadding stage } \\
\text { after } 5 \text { years }(0 / \\
\text { I/II/III/IV) }\end{array}$ & $\begin{array}{c}(\mathrm{N}=25) 5 / 2 / 4) \\
6 / 8(20 / 8 / 16) \\
24 / 32)\end{array}$ & $\begin{array}{c}(\mathrm{N}=16) 3 / 1 / 4 / \\
2 / 6(19 / 6 / 25 / \\
13 / 38)\end{array}$ & 0.878 & $\begin{array}{c}(\mathrm{N}=34), 7 / 2 / \\
8 / 7 / 10(21 / 6) \\
24 / 21 / 29)\end{array}$ & $\begin{array}{c}(\mathrm{N}=7), 1 / 1 / 0 / \\
1 / 414 / 14 / 0 / \\
14 / 57\end{array}$ & 0.441 \\
\hline \multicolumn{7}{|c|}{$\begin{array}{l}\text { Data are presented as mean } \pm \text { SD or } \mathrm{n}(\%) \text {, unless otherwise stated. Chest radiographs to determine } \\
\text { Scadding stages were missing from three sarcoidosis patients at diagnosis, from } 13 \text { patients } 2 \text { years after } \\
\text { diagnosis and from } 35 \text { patients } 5 \text { years after diagnosis. Scadding stages: } 0 \text { : Normal chest radiograph; I: } \\
\text { BHL; II: BHL with pulmonary infiltrates; III: pulmonary infiltrates without BHL; IV: fibrosis. SFN: Small } \\
\text { fibre neuropathy, PAB: P. acnes-specific monoclonal antibodies that react with cell membrane-bound } \\
\text { lipoteichoic acid; BHL: bilateral hilar lymphadenopathy. }{ }^{\#}: \text { p-values regarding age was calculated using an } \\
\text { independent samples t-test. Other p-values were calculated using a Fisher's exact test, except for sex } \\
\text { (both p-values), extrapulmonary involvement in the } P \text {. acnes score group, skin, heart and nerve system } \\
\text { involvement in the } P \text {. acnes score group and Scadding stages; }{ }^{\uparrow} \text { : Age at time of biopsy. }\end{array}$} \\
\hline
\end{tabular}

A limitation of the study was that the COS after 5 years could not be determined in all patients. However, as the proportion of patients within COS group A and B was quite similar after 2 and 5 years and also the results regarding association with $P$. acnes staining, we assume that this analysis on a smaller group of patients has not introduced a bias. A disadvantage of using COS is the fact that disease status and medication use have been retrospectively assessed. As we only scored whether medication was used or not, we have no information on patients who declined the use of medication while they actually needed it.

Another limitation is that we probably have a more severe patient group than other general hospitals, as the St Antonius Hospital is a national referral centre for interstitial lung disease and sarcoidosis. As a consequence, we had very few patients in the resolved and minimal disease COS groups. For this reason, we did not have enough patients in every group to adequately analyse whether disease status alone, irrespective of use of medication, was associated with presence of $P$. acnes in tissue and granulomas. However, instead of disease status we think that need for treatment could be of more value regarding the presence of $P$. acnes. If we want to further explore the use of antibacterial therapy, in our opinion, it is more relevant to focus on patients who actually need treatment.

In future studies, it would be interesting to add a second detection method, such as PCR, to the immunostaining. To accurately compare the results, development and use of a primer specific to LTA of the $P$. acnes bacteria would be valuable.

To conclude, this study confirms the presence of $P$. acnes in tissues and granulomas in $40 \%$ and $17 \%$, respectively of Dutch patients with sarcoidosis. Interestingly, the presence of $P$. acnes inside granulomas of 
Dutch sarcoidosis patients was associated with a chronic disease phenotype and requirement of treatment. Regardless of whether the role of $P$. acnes is antigenic or mitogenic, our data contribute to the premise that it is relevant to further explore antibacterial therapy as a treatment option for a subset of sarcoidosis patients.

Author contributions: E. Beijer contributed to data acquisition and analysis, statistical analysis, and drafting and editing of the manuscript. K. Seldenrijk participated in the design of the study, scored the staining results and edited the manuscript. Y. Eishi developed the PAB antibody, advised on study design and edited the manuscript. K. Uchida reviewed the methods and advised on the automated staining protocol. J. Damen carried out all the immunohistochemical staining and developed the automated staining protocol. J.C. Grutters supervised the project and edited the manuscript. M. Veltcamp designed the study, supervised the project and edited the manuscript.

Support statement: This study is part of the TopZorg grant funded by ZonMw (number 10070012010004). This funder had no involvement in the study design; in the collection, analysis and interpretation of the data; in the writing of the report; or in the decision to submit the paper for publication. Funding information for this article has been deposited with the Crossref Funder Registry.

Conflict of interest: None declared.

\section{References}

Ramachandraiah V, Aronow W, Chandy D. Pulmonary sarcoidosis: an update. Postgrad Med 2017; 129: 149-158. Spagnolo P, Rossi G, Trisolini R, et al. Pulmonary sarcoidosis. Lancet Respir Med 2018; 6: 389-402. Baughman RP, Lower EE. Treatment of sarcoidosis. Clin Rev Allergy Immunol 2015; 49: 79-92.

4 Esteves T, Aparicio G, Garcia-Patos V. Is there any association between sarcoidosis and infectious agents?: a systematic review and meta-analysis. BMC Pulm Med 2016; 16: 165.

5 Zhou Y, Wei YR, Zhang Y, et al. Real-time quantitative reverse transcription-polymerase chain reaction to detect propionibacterial ribosomal RNA in the lymph nodes of Chinese patients with sarcoidosis. Clin Exp Immunol 2015; 181: 511-517.

6 Ebe Y, Ikushima S, Yamaguchi T, et al. Proliferative response of peripheral blood mononuclear cells and levels of antibody to recombinant protein from Propionibacterium acnes DNA expression library in Japanese patients with sarcoidosis. Sarcoidosis Vasc Diffuse Lung Dis 2000; 17: 256-265.

7 Yorozu P, Furukawa A, Uchida K, et al. Propionibacterium acnes catalase induces increased Th1 immune response in sarcoidosis patients. Respir Investig 2015; 53: 161-169.

8 Schupp JC, Tchaptchet S, Lutzen N, et al. Immune response to Propionibacterium acnes in patients with sarcoidosis--in vivo and in vitro. BMC Pulm Med 2015; 15: 75-77.

9 Eishi Y. Etiologic link between sarcoidosis and Propionibacterium acnes. Respir Investig 2013; 51: 56-68.

10 Ishige I, Eishi Y, Takemura T, et al. Propionibacterium acnes is the most common bacterium commensal in peripheral lung tissue and mediastinal lymph nodes from subjects without sarcoidosis. Sarcoidosis Vasc Diffus lung Dis 2005; 22: 33-42.

11 Zhao MM, Du SS, Li QH, et al. High throughput 16SrRNA gene sequencing reveals the correlation between Propionibacterium acnes and sarcoidosis. Respir Res 2017; 18: 28.

12 Ishige I, Usui Y, Takemura T, et al. Quantitative PCR of mycobacterial and propionibacterial DNA in lymph nodes of Japanese patients with sarcoidosis. Lancet 1999; 354: 120-123.

13 Eishi Y, Suga M, Ishige I, et al. Quantitative analysis of mycobacterial and propionibacterial DNA in lymph nodes of Japanese and European patients with sarcoidosis. J Clin Microbiol 2002; 40: 198-204.

14 Furusawa H, Suzuki Y, Miyazaki Y, et al. Th1 and Th17 immune responses to viable Propionibacterium acnes in patients with sarcoidosis. Respir Investig 2012; 50: 104-109.

15 Beijer E, Kraaijvanger R, Roodenburg C, et al. Simultaneous testing of immunological sensitization to multiple antigens in sarcoidosis reveals an association with inorganic antigens specifically related to a fibrotic phenotype. Clin Exp Immunol Sci Progr 2021; 1: 115-124.

16 Teixeira D, Ishimura ME, de Souza Apostólico J, et al. Propionibacterium acnes enhances the immunogenicity of hivbr18 human immunodeficiency virus-1 vaccine. Front Immunol 2018; 9: 177.

17 Ghaffar A, Cullen RT, Woodruff MFA. Further analysis of the anti-tumour effect in vitro of peritoneal exudate cells from mice treated with Corynebacterium parvum. Br J Cancer 1975; 31: 15-24.

18 Keller R, Keist R, van der Meide PH. Modulation of tumoricidal activity, induced in bone-marrow-derived mononuclear phagocytes by interferon $\gamma$ or Corynebacterium parvum, by interferon $\beta$, tumor necrosis factor, prostaglandin E2, and transforming growth factor $\beta$. Int J Cancer 1991; 49: 796-800.

19 Ananias RZ, Rodrigues EG, Braga EG, et al. Modulatory effect of killed Propionibacterium acnes and its purified soluble polysaccharide on peritoneal exudate cells from $\mathrm{C} 57 \mathrm{Bl} / 6$ mice: major NKT cell recruitment and increased cytotoxicity. Scand J Immunol 2007; 65: 538-548.

20 Negi M, Takemura T, Guzman J, et al. Localization of Propionibacterium acnes in granulomas supports a possible etiologic link between sarcoidosis and the bacterium. Mod Pathol 2012; 25: 1284-1297.

21 Vorselaars AD, Crommelin HA, Deneer VH, et al. Effectiveness of infliximab in refractory FDG PET-positive sarcoidosis. Eur Respir J 2015; 46: 175-185.

22 Costabel U, Hunninghake GW. ATS/ERS/WASOG statement on sarcoidosis. Sarcoidosis Statement Committee. American Thoracic Society. European Respiratory Society. World Association for Sarcoidosis and Other Granulomatous Disorders. Eur Respir J 1999; 14: 735-737.

23 Baughman RP, Nagai S, Balter M, et al. Defining the clinical outcome status (COS) in sarcoidosis: results of WASOG Task Force. Sarcoidosis Vasc Diffus Lung Dis 2011; 28: 56-64.

24 Yeon SJ, Matsumoto SE, Yamashita M, et al. Propionibacterium acnes acts as an adjuvant in in vitro immunization of human peripheral blood mononuclear cells. Biosci Biotechnol Biochem 2007; 71: 1963-1969. 
25 Mussalem JS, Squaiella-Baptistão CC, Teixeira D, et al. Adjuvant effect of killed Propionibacterium acnes on mouse peritoneal B-1 lymphocytes and their early phagocyte differentiation. PLoS ONE 2012; 7: e33955.

26 Takemori N, Nakamura M, Kojima M, et al. Successful treatment in a case of Propionibacterium acnes-associated sarcoidosis with clarithromycin administration: a case report. J Med Case Rep 2014; 8: 15.

27 Inoue Y, Teraki Y. Association of Propionibacterium acnes with the efficacy of minocycline therapy for cutaneous sarcoidosis. Int J Dermatol 2020; 59: 704-708.

28 Ishibashi K, Eishi Y, Tahara N, et al. Japanese antibacterial drug management for cardiac sarcoidosis (J-ACNES): a multicenter, open-label, randomized controlled study. J Arrhythmia 2018; 34: 520-526. 